\title{
Mass-geometric parameters improvement of gearbox by using vibration analysis
}

\author{
Viktoriia ${\text { Batizi }{ }^{1,}, \text { Dmitriy Likhachev }}^{2}$ \\ ${ }^{1}$ Design Engineer of Car bodies and Cabines Department, FSUE «NAMI», Moscow 125438, Russian \\ Federation \\ ${ }^{2}$ Lead Design Engineer of Transmission Department, PhD, FSUE «NAMI», Moscow 125438, \\ Russian Federation
}

\begin{abstract}
Mass-geometric parameters of transmission elements have a big impact on the cost of the car in whole. A large number of automakers compete for a reduction in prices for manufactured products and are eager to facilitate finished products. In addition, the durability of the car and its comfort, in particular, the noise level, are largely determined by vibration loads, the reduction of which becomes more important. The relevance of the study is the need to improve the design of the planetary gearbox at the stages of finishing the car in order to reduce its weight and ensure the after resonance operation modes of its components.
\end{abstract}

\section{Introduction}

The mandatory requirement of the market is the optimization of the product, which makes it possible to obtain the most light and technological design.

The purpose of the work is to develop a methodology for improvement of the massgeometric parameters of a planetary gearbox based on the evaluation of the stress-strain state of its parts and the modal analysis of the conditions of the above resonance operating modes.

Automated engineering calculations are used to solve the problem. Computer simulation of the gearbox elements will help to check the correctness of the adopted technical solutions and eliminate the need to produce a large number of prototypes.

Practical value consists in developing a method that allows to improve the massgeometric parameters of the elements of the planetary gearbox, which will allow to obtain at the development stage of the car the optimal, from the point of view of strength, manufacturability and vibration loading, a design that has a comparatively small mass in comparison with the original prototype.

\footnotetext{
*Corresponding author: viktoriia.batizi@nami.ru,dmitriy.likhachev@nami.ru
} 


\section{Purpose and scope of the test object}

The object of the study is a planetary 9-speed automatic transmission (A/T) of a vehicle with a hybrid drive, developed at the SRC RF FSUE "NAMI". The automatic transmission is designed for installation on executive cars.

Involute splined joints are used to connect the parts and transmit torque in a design that can take significant loads with relatively small joint sizes. They are also the most technologically advanced in terms of series production and mass production.

In the planetary mechanism, the axial forces from the gears are perceived by thrust needle bearings. This type of bearings is able to perceive significant axial forces with small dimensions.

The planetary mechanism is a three-dimensional frame consisting of two rings (cheeks) connected by equally spaced beams. Cheeks can be mated to the neck of the support shaft of the carrier or to the flange for its attachment in the transmission housing (Fig.1).

The thrust rings, needle bearings and sliding bearings included in this design are not taken into account to simplify the design model.

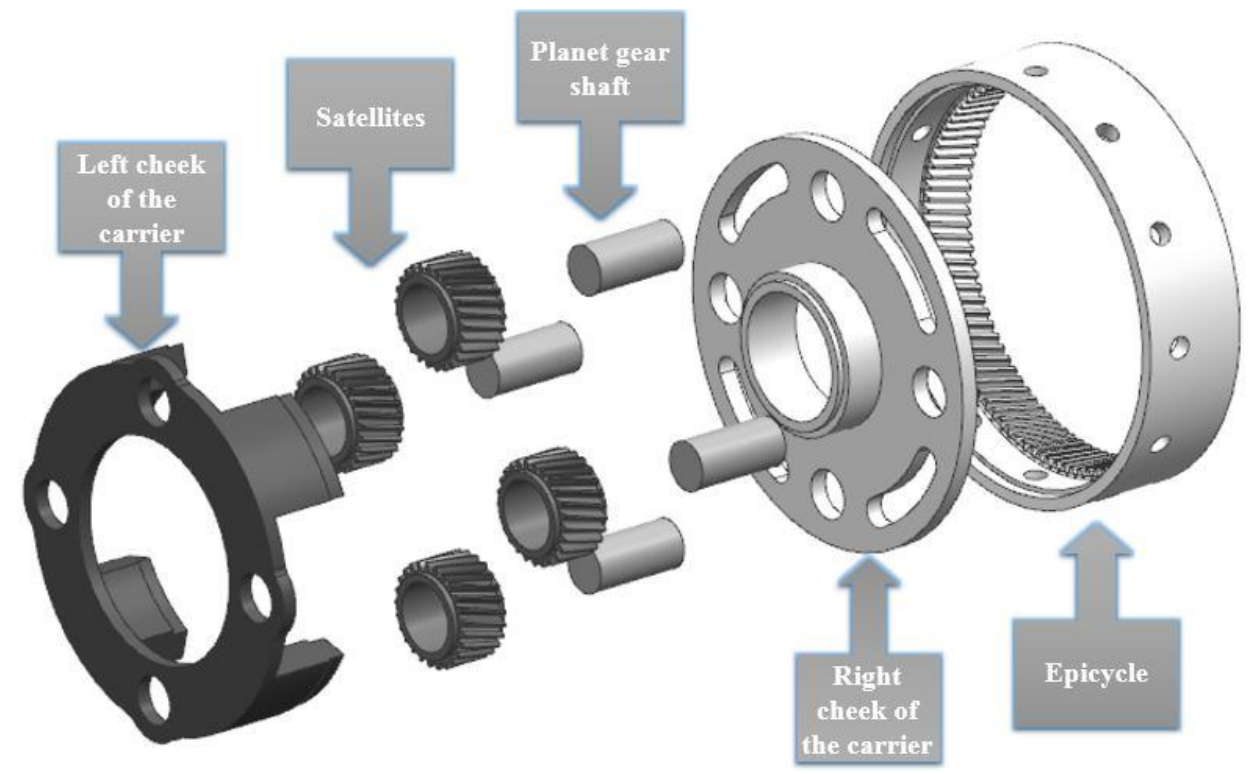

Figure 1. Object of research: planetary gear set

\section{Research by using FEM}

As an example of carrying out virtual experiments, the critical loads are calculated, in consequence of which there is a destruction of the elements of the planetary gear of automatic transmission (satellites, central and crown gears).

Weight, stress, displacement, natural frequencies and other characteristics are considered as objective functions and limitations, which makes it possible to analyze mechanical systems and evaluate the functionality of the product in whole, its individual components and details of the mechanisms.

In the scientific work the following tasks are solved:

- determination of the stress-strain state of planetary gear taking into account the dimensional application of the load;

- determination of frequencies and natural modes of vibrations; 
- construction of a frequency Campbell diagram for gear wheels (satellites);

- analysis of the obtained results.

\subsection{Calculation of the stress-strain state of gearing}

The purpose of the calculation is to determine the stiffness of the planetary mechanism model and calculating max equivalent stress in the most loaded areas of the construction under static load applications.

According to the calculation results, it is found that the values of the equivalent stresses in the epicyclic gear region exceed the yield stress of $\sigma_{02}$ by $100 \mathrm{MPa}$, and on the tooth contour of the satellite by $47 \mathrm{MPa}$ (Fig. 2).

Thus, it is necessary to change the geometric parameters of the gear wheels, which in turn will affect the change in the dimensions and mass of the elements of the planetary mechanism. The design of the planetary gear should be improved in such a way that when the calculation is repeated, the maximum values of the operating stress do not exceed the yield strength.

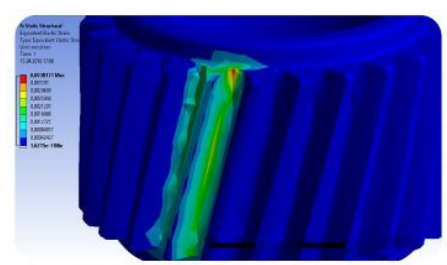

Equivalent Elastic Strain

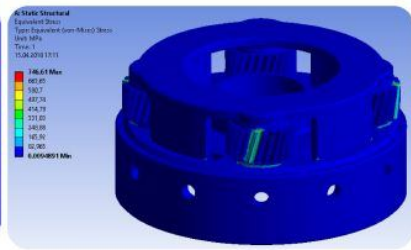

Equivalent Stress (von-Mises), MPa Maximum Principal Stress $\left(\sigma_{1}\right)$, MPa

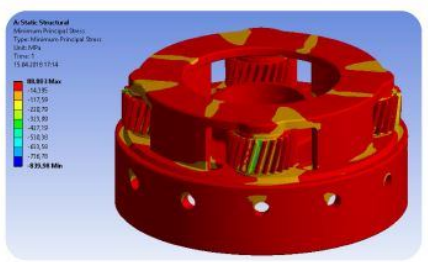

Minimum Principal Stress $\left(\sigma_{3}\right)$, MPa

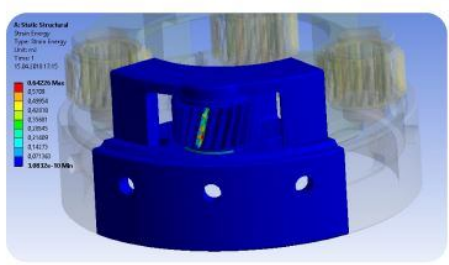

Strain Energy

Figure 2. Distribution of strain and equivalent stresses

However, before optimizing the design, in order to achieve maximum safety factor, it is necessary to build a resonance-frequency diagram.

When analyzing the stress state of a structure for stress tensors, the eigenvalues determine the principal normal stresses, and the directions associated with the triple of values of the principal stresses are given by the eigenvectors [1].

In the dynamic analysis of mechanical systems, for example, in modal analysis of vibrations, the eigenvalues correspond to the natural frequencies of oscillations, and the eigenvectors characterize the deformation of the structure corresponding to each frequency of natural oscillations. When performing the resonance-frequency calculation of designs, the eigenvalues allow to determine the critical loads, the excess of which leads to deformation or destruction $[2,3]$. 


\subsection{Method for estimating the natural oscillation frequencies of planetary gear set.}

The next task is to determine the natural oscillation frequencies of the planetary gear set. Let us take a brief look at fundamental principles used in the calculation of the natural oscillations of the design. Equation of oscillations for a system with several degrees of freedom with free vibrations (i.e. without external load and damping) (1):

$$
[K]-\omega^{2}[M]\{\varphi\}=0,
$$

where $[K]$ - stiffness matrix;

$[M]$ - matrix of inertia moment;

$\omega$ - natural frequencies;

$\varphi$ - oscillation modes.

Equations of natural oscillations is a particular case of the equation of motion (2):

$$
[M] \cdot\left\{\varphi^{\prime \prime}\right\}+[K] \cdot\{\varphi\}=0,
$$

where $\varphi$ - angular displacement, [rad];

$\varphi^{\prime \prime}-$ angular acceleration, $\left[\mathrm{rad} / \mathrm{s}^{2}\right]$.

The procedure for analyzing the calculation of the forms and natural oscillation frequencies is reduced to processing the results of modal analysis (calculation of eigenmodes and vibration frequencies at different operating modes) and constructing a resonancefrequency diagram for planetary gears (satellites).

The design model of the compound gear of planetary gear set was built by using the ANSYS Workbench v.17.2 preprocessor. The compound gear consists of a satellite and a crown of a gear (epicycle).

In the capacity of a visual display and analysis of the results is the Campbell diagram the dependence of the value of the natural frequency on the epicycle rotation frequency. Calculations are performed at the following rotational speeds of the wheel (in revolutions per minute): $0 \%, 25 \%, 50 \%, 75 \%, 100 \%$ of the rotation speed of the compound gear. Accordingly, the dependencies were constructed according to the five operating modes.

The axis of abscissas of the ray-diagram represents the rotation speed of the engine and along the ordinate axis the frequencies of the perturbing factor of the satellite. The graph is plotted with rays corresponding to the perturbing factors in the transmission and the natural frequencies of the system in the form of lines parallel to the abscissa. The intersection points of these two groups of rays determine the resonant rotational velocities.

Gear sets operate with a wide variation of the angular velocities of the gear wheels, which leads to a corresponding displacement of the spectrum of the perturbing forces on the frequency axis [4].

To determine the rotational frequency with respect to each mode of operation, we use the following formula (3):

$$
n_{i}=\left(n_{\max } \cdot k\right) / 60[r p m]
$$

where $n_{i}$-rate speed relative to $i$-th mode of operation;

$n_{\max }$ - maximum rate speed; 
$k$ - coefficient corresponding multiplicity of vibration modes.

To determine the rotation frequencies of a satellite with a certain mode and its nodal diameter, the following formula (4) is used:

$$
n_{\text {satellite }}=\frac{f_{i} \cdot 60}{z_{\text {epicycle }}}[\mathrm{rpm}] \text {, }
$$

where $n_{\text {satellite }}$ - rotational velocity of the satellite with a certain mode and its nodal diameter; $f_{i}$ - natural oscillation frequency;

$z_{\text {epicycle }}-$ quantity of epicycle teeth.

After the spectrum of the natural frequencies of the system is determined, a Campbell diagram must be constructed for all forms of oscillations of the satellite. As an example, Fig. 3 shows a diagram for five natural oscillation modes of the satellite at $0 \%$ of the load.

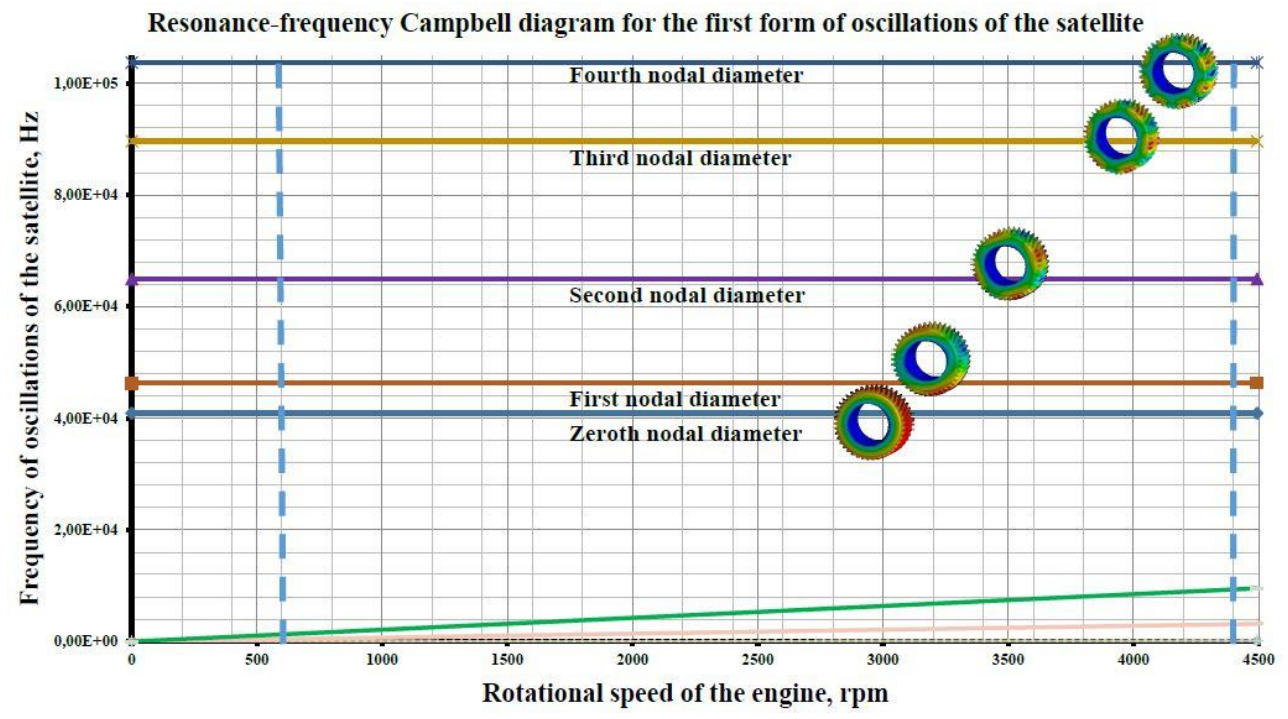

Figure 3. Resonance-frequency Campbell diagram for the first form of oscillations of the satellite

By analyzing the diagram it can be concluded that there is no intersections of the natural oscillation frequencies with the lines of the exciting harmonics (the same conclusion applies to the other load conditions). This suggests that the Campbell diagram did not reveal any possible resonances, so the absence of dangerous vibration frequencies does not affect the stress-strain state of the satellite.

It is also worth noting that the oscillations are beyond the range of engine operation $(600 \ldots 4494 \mathrm{rpm})$ and are high-frequency. In this case, the parameter of the moment of inertia $J$ can be used as the occurrence of resonant modes of assessment criterion, because the highfrequency range is determined by its low value, [5]:

$$
J=m \cdot r^{2}
$$

where $m$ - mass, $[\mathrm{kg}]$;

$r$-radius (gear wheel), [mm]. 
It should be taken into account that when changing the geometrical parameters of the gearing, for example, increasing the radius or mass it will also increase the moment of inertia. In turn, this will lead to a decrease in the natural frequencies, which can fall on the perturbation range.

\section{Results of the research work}

In this paper, we propose a method of estimating the frequencies of natural vibrations for the planetary gear set of satellites. Flexure-torsional coupling in the general case is non-linear and determines the interaction of the components of the oscillations with the operating mode of the gears, taking into account the centrifugal forces.

The advantage of this method is that there is no need to rebuild geometric models and design grids at each step of the calculation, which leads to a significant reduction in labor intensity.

The method will allow to predict and estimate the level of vibration stresses of the vital parts of the planetary gear sets.

Based on the results of solving this problem, the levels of the stressed-deformed state of the operating satellites are determined.

It was found that the resonance regimes depend on the change in geometric parameters and mass $m$. The decrease in mass makes it possible to increase the natural frequency $\omega_{0}$ so that the planetary gear set will predeterminedly work in the above resonance mode $\left(\omega>\omega_{0}\right)$, thereby increasing the risk of growth of the calculated stress-strain state and lead to the destruction of the part. The increase in the mass of the gears, in particular, will lead to the growth of the safety factor, however, from the point of modal analysis, the gears will operate in the below resonance frequency band $\left(\omega<\omega_{0}\right)$.

The detuning from the resonant modes must go in parallel with the calculation of the stress-strain state of the components of the planetary gear sets, thereby avoiding the occurrence of undesirable rotational velocities, evaluating the effectiveness of changing the inertial-rigid parameters of transmission.

\section{References}

[1] N.W.M. Bishop, F. Sherratt Finite Element Based Fatigue Calculations, NAFEMS, (2000)

[2] K. -J. Bathe Finite Element Procedures, Prentice Hall, (1996)

[3] L. Efremov Computerized Analyses of Torsional Vibrations in Propulsion Units: Theory and Practice. - St.-Petersburg: Nauka, (2007)

[4] A. Alshyyab, A. Kahraman A non-linear dynamic model for planetary gear sets. J MutiBody Dyn 221(4), (2007)

[5] Tutorial. Comp: V.P. Rzhevsky, F.V. Parovay, D.S. Lezhin, V.S. Melentiev, A.S. Gvozdev - Samara: Samara Publishing House. University, (2011) 